\title{
Herausragende Wissenschaftspreise und Ehrungen für pädiatrische Onkologen
}

\section{Outstanding Awards and Honors for Pediatric Oncologists}

T. Klingebiel

Bibliografie

DOI http://dx.doi.org/ $10.1055 / \mathrm{s}-0032-1309034$ Klin Padiatr 2012; 224: 121-123 (c) Georg Thieme Verlag KG Stuttgart · New York ISSN 0300-8630

Korrespondenzadresse

Prof. Dr. Thomas Klingebiel

Zentrum für Kinder- und Jugendmedizin

Klinik III

Klinikum der Johann-WolfgangGoethe-Universität Frankfurt am Main

Theodor-Stern-Kai 7 60590 Frankfurt am Main Tel.: +49/69/6301 5094 Fax: $+49 / 69 / 63016700$ thomas.klingebiel@kgu.de
Wissenschaftliche Preise und Ehrungen sind etwas Wunderbares. Zuerst und vor allen Dingen natürlich für die Ausgezeichneten selbst, deren wissenschaftliche Leistungen vor einem sachund fachkundigen Publikum die ihr gebührende Anerkennung erfahren. Sie lenken allerdings auch den Blick der Öffentlichkeit und der Scientific Community auf die Fachgesellschaft, der die Ausgezeichneten angehören. Häufen sich solche Ehrungen und Auszeichnungen in kurzer Zeit, kann mit einem gewissen Recht davon ausgegangen werden, dass auf die Arbeit der Fachgesellschaft selbst etwas von dem Glanz der Ausgezeichneten abstrahlt. So ist es der Gesellschaft für Pädiatrische Onkologie und Hämatologie (GPOH) in den letzten Wochen und Monaten ergangen, da eine stattliche Anzahl ihrer Mitglieder in den Genuss wichtiger Preise und Ehrungen kamen.

Professor Klaus-Michael Debatin erhielt im Januar 2012 aus den Händen von Fritz Pleitgen als Präsident der Deutschen Krebshilfe den Deutschen Krebshilfe Preis (gemeinsam mit Professor Dr. Peter Krammer, Deutsches Krebsforschungszentrum Heidelberg) für seine wegweisenden Arbeiten auf dem Gebiet der Apoptose-Forschung. Professor Debatin ist seit 1997 Ärztlicher Direktor der Universitätsklinik für Kinder- und Jugendmedizin Ulm, 2010 wurde er zum Vizepräsidenten für Medizin der Universität Ulm ernannt, und er war auch der langjährige Dekan des Fachbereichs Medizin. In ihrer Laudatio sagte Frau Professor Charlotte Niemeyer, dass die Deutsche Krebshilfe mit Professor Debatin einen Wissenschaftler auszeichnet, der die „Apoptoseforschung in Deutschland erheblich vorangebracht und insbesondere zur klinischen Anwendung geführt hat". Die wegweisenden Arbeiten von Prof. Debatin aus den letzten Jahren wurden in höchstrangigen Fachzeitschriften wie Cancer Cell, Journal of Experimental Medicine, Blood und Cell publiziert $[7,8,11,22,24,25,29,31]$. Prof. Debatin ist Mitglied der Deutschen Akademie der Naturforscher Leopoldina - Nationale Akademie der Wissenschaften, Mitglied der Heidelberger Akademie der Wissenschaften und auch schon vorher Träger mannigfaltiger Preise und Auszeichnungen.

Es ist gewiss kein Zufall, dass seine Laudatorin Professorin Charlotte Niemeyer kurze Zeit später den Deutschen Krebspreis 2012 der Deutschen Krebsgesellschaft aus den Händen von Professor Hohenberger entgegennehmen konnte. Professorin Niemeyer ist Ärztliche Direktorin der Klinik für Pädiatrische Onkologie und Hämatologie des Zentrums für Kinder- und Jugendmedizin der Albert-Ludwigs-Universität Freiburg, war über $3 \frac{1}{2}$ Jahre Prorektorin der Universität und ist gegenwärtig Exzellenzbeauftragte des Rektors der Universität. Auch sie ist Mitglied der Deutschen Akademie der Naturforscher Leopoldina Nationale Akademie der Wissenschaften und Mitglied zahlreicher nationaler und internationaler Fachgesellschaften. Sie wurde ausgezeichnet für ihre translationalen Arbeiten auf dem Gebiet der Erforschung von kongenitalen Knochenmarkerkrankungen, insbesondere der juvenilen myelomonozytären Leukämie und der Übertragung ihrer Erkenntnisse aus dem Labor in klinische Studien. Sie hat führend die Internationale Working Group für Myelodysplastische Erkrankungen im Kindesalter aufgebaut und mehrere klinische Studien, die wesentlich zum Erkenntnisgewinn beigetragen haben, auf den Weg gebracht. Ihre Arbeiten, für die sie ausgezeichnet wurde, wurden in Nature Genetics, Nature, Blood und Oncogene veröffentlicht $[9,16,20,26,30]$.

Kurz vor Jahresende erhielt Professor Alfred Reiter (gemeinsam mit Professorin Catherine Patte, Paris) den SIOP-Europe Life Time Achievement Award für sein Lebenswerk. Der Preis wurde in Stockholm auf der ECCO-Jahrestagung vergeben und für lebenslange klinische Forschungsarbeiten auf dem Gebiet der Non-Hodgkin Lymphome verliehen, die Professor Reiter zur internationalen Sichtbarkeit und Anerkennung geführt hat. Professor Reiter ist Direktor der Kinderklinik Pädiatrische Hämatologie und Onkologie der Justus-Liebig Universität Gießen und leitet seit mehr als zwanzig Jahren die NHL-BFM-Studiengruppe. Seine Arbeit hat maßgeblich zum dramatischen Anstieg der Überlebenswahrscheinlichkeit von Kindern und Jugendlichen mit NonHodgkin-Lymphomen in Europa und vermutlich weltweit beigetragen. Sie ist ein Beispiel für die unglaubliche Veränderung, die die pädiatrische Onkologie in den letzten Jahren erfahren hat $[4,5,23,27,32]$.

Eine Auszeichnung auf hohem internationalem Niveau hat Professor Christoph Klein im November 2011 auf dem ASH-Meeting in San Diego entgegennehmen können. Professor Klein ist Direktor der Universitätskinderklinik der LudwigMaximilians-Universität München und erhielt den William Dameshek Preis für seine grundlegenden Beiträge zur Hämatologie. Wegweisend sind seine Entdeckungen der Mutationen von HAX1-Genen bei Patienten mit schwerer kongenitaler Neutropenie und seine Entdeckung der humanen P14/ROBLD3- und G6PC3-Defizienz. Auch diese Defekte führen zu schweren kongenitalen Neutropenien. Der William Dameshek Preis ist benannt nach dem ersten Herausgeber von Blood und früheren Präsidenten der American 
Society of Hematology. Der Preis wird an Forscher verliehen, die jünger als 50 Jahre sind und herausragende Beiträge zur Hämatologie geleistet haben. Professor Klein nahm den Preis aus den Händen des ASH-Präsidenten J. Evan Sadler entgegen, der in seiner Laudatio sagte, dass dieser Preis „for his pioneering research into the pathophysiology of rare diseases which has had a direct and profound impact on clinical medicine" verliehen wird. Professor Klein und sein Team haben in den letzten Jahren erhebliche Beiträge zur Entdeckung von Mutationen geleistet, die z.B. den Interleukin-10-Rezeptor betreffen und mit der „severe early-onset inflammatory bowel disease“ kombiniert sind. Ein zweiter kürzlicher Beitrag war, die Behandlung von WiskottAldrich-Syndrom-Patienten im Rahmen eines Gentherapieprogramms. Arbeiten von Professor Klein wurden in zahlreichen Fachzeitschriften wie New England Journal of Medicine, Nature Medicine, Nature Genetics, Immunity und Journal of Experimental Medicine publiziert $[1-3,12,28]$. Schon vorher hat er renommierte Preise wie den Adalbert-Czerny Preis und den GlaxoSmithKline Award erhalten. 2010 war er der erste Pädiater, der den Gottfried-Wilhelm-Leibniz Preis der Deutschen Forschungsgemeinschaft entgegennehmen konnte.

Eine Auszeichnung besonderer Art ist Professorin Simone Fulda zuteil geworden: Professorin Fulda ist Direktorin des Instituts für Experimentelle Tumorforschung in der Pädiatrie der JohannWolfgang-Goethe-Universität Frankfurt am Main. Auch sie hat sich als frühere Mitarbeiterin von Professor Debatin einen Namen mit der Apoptoseforschung gemacht und in vielen Beiträgen nachweisen können, warum das Apoptoseprogramm bei Krebszellen nicht mehr funktioniert. Diese Beiträge münden mittlerweile in erste medikamentöse Behandlungen, um das Apoptoseprogramm wieder in Gang zu bringen [8,10,11,21,22,31]. Für diese Beiträge hat sie bereits viele nationale und internationale Preise erhalten, u.a. war sie Heisenberg-Stipendiatin der DFG. Sie ist in zahlreichen internationalen Verbundforschergruppen tätig wie der DFG Klinischen Forschergruppe „Apoptoseregulation und ihre Störungen bei Krankheiten“, im Marie Curie Research Training and Network „Apop Train“(gefördert durch die EU FP6), BMBF-Netzwerk „Apoptose als Target in der Tumortherapie“ im TranSarNet des BMBF und im KOSA-Netzwerk der Deutschen Krebshilfe. Trotz allem ist es noch eine besondere Anerkennung und Auszeichnung ihrer Arbeit, dass sie durch den Bundespräsidenten kürzlich als ordentliches Mitglied in den Wissenschaftsrat berufen wurde.

Der Wissenschaftsrat ist eines der wichtigsten wissenschaftspolitischen Beratungsgremien in Deutschland. Er berät die Bundesregierung und die Regierung der Länder in allen Fragen der inhaltlichen und strukturellen Entwicklung der Wissenschaft und in der Forschung des Hochschulbereichs. Es gehört auch zu seinen Aufgaben zur Sicherung der internationalen Konkurrenzfähigkeit der Wissenschaft in Deutschland im nationalen und europäischen Wissenschaftssystem beizutragen (www.wissen schaftsrat.de). Er gibt Empfehlungen und Stellungnahmen zu den Aufgabenfeldern der Wissenschaftspolitik, insbesondere zu den wissenschaftlichen Institutionen (Universitäten, Fachhochschulen, außeruniversitäre Einrichtungen) und zu übergreifenden Fragen des Wissenschaftssystems. Der Wissenschaftsrat hat 24 berufene wissenschaftliche Mitglieder, von denen 4 Mediziner sind. Mitglied in einem solchen Gremium zu sein, bedeutet Ehrung und Herausforderung zugleich. Professorin Fulda wird also in einer ganz zentralen Schaltstelle der deutschen Wissenschaftspolitik tätig sein. Dort eine Vertreterin der Pädiatrie und insbesondere der pädiatrischen Onkologie zu wissen, ist für uns wichtig.

Diese letzten Monate mögen für die pädiatrische Onkologie besonders „fruchtbare Monate“ gewesen sein. Allerdings zeigt sich in der großen Anerkennung, die die 5 Mitglieder unserer Fachgesellschaft erfahren haben und zu der wir ganz herzlich im Namen aller unserer Mitglieder gratulieren, auch die wissenschaftliche Leistungsfähigkeit und den wissenschaftlichen Stellenwert der Arbeit der pädiatrischen Onkologie in den letzten Jahren und Jahrzehnten. Diese Ehrungen sind für die Geehrten eine große Anerkennung ihrer Leistungen, für uns als Fachgesellschaft Anerkennung und Herausforderung gleichzeitig.

Für den Erfolg der Exponenten der GPOH sprechen neben der herausragenden persönlichen Expertise der Forscher und ihres unmittelbaren Umfelds die wissenschaftliche Aufgeschlossenheit und die vielfältigen Fördermöglichkeiten. Alle vier Faktoren - Forscherpersönlichkeit, Umfeld und Kooperation, Aufgeschlossenheit und Mitarbeiter, Fördermöglichkeiten - sind notwendige Bedingungen für derartige Erfolge, ohne sie jedoch zu garantieren. Einen wesentlichen Beitrag hat sicher auch die Förderung durch die Bundesministerien, die Deutsche Krebshilfe [13], die deutsche Kinderkrebsstiftung, die lokalen Elternvereine, die Kompetenznetzförderung [6] und die Kind-Philipp-Stiftung [19] geleistet. Diese Förderer [17] haben über einen langen Zeitraum die besonderen Belange der pädiatrischen Onkologie und Hämatologie und deren Auseinandersetzung mit seltenen Erkrankungen $[14,15,18]$ erkannt und berücksichtigt und verdienen besonderen Dank. Den Preisträgern möchten wir weitere Erfolge, Gesundheit und Schaffenskraft zum Wohle unserer Patienten und unserer gemeinsamen Anliegen wünschen. Uns allen in der $\mathrm{GPOH}$ mögen diese Ehrungen Ansporn und Verpflichtung zugleich sein.

\section{Literatur}

1 Boztug K, Appaswamy G, Ashikov A et al. A syndrome with severe congenital neutropenia and mutations in G6PC3. New Engl J Med 2009; 360: 32-43

2 Boztug K, Schmidt M, Schwarzer A et al. Gene therapy for Wiskott Aldrich Syndrome. New Engl J Med 2010; 363: 1918-1927

3 Boztug K, Xiao-Qi Ding XD, Hartmann $H$ et al. HAX1 mutations causing SCN and neurological disease lead to cerebral microstructural abnormalities documented by quantitative MRI. Am J Med Genet 2010; 152A: 3157-3166

4 Brugières L, Le Deley MC, Rosolen A et al. Impact of the methotrexate administration dose on the need for intrathecal treatment in children and adolescents with anaplastic large-cell lymphoma: results of a randomized trial of the EICNHL Group. J Clin Oncol 2009; 27: 897-903

5 Burkhardt B, Oschlies I, Klapper W et al. Non-Hodgkin's lymphoma in adolescents: experiences in 378 adolescent NHL patients treated according to pediatric NHL-BFM protocols. Leukemia 2011; 25: 153-160

6 Creutzig $U$, Herold R, Henze G. Results of the Competence Net Pediatric Oncology and Haematology - a view back. Klin Padiatr 2010; 222: 333-336

7 Debatin K-M. Chronic lymphocytic leukemia: Keeping cell death at bay. Cell 2007; 129: 853-855

8 Fakler M, Loeder $S$ Vogler et al. Small molecule XIAP inhibitors cooperate with TRAIL to induce apoptosis in childhood acute leukemia cells and overcome Bcl-2-mediated resistance. Blood 2009; 113: $1710-1722$

9 Flotho C, Steinemann D, Mullighan CG et al. Genome-wide single nucleotide polymorphism analysis in juvenile myelomonocytic leukemia identifies uniparental disomy surrounding the NF1 locus in cases associated with neurofibromatosis but not in cases with mutant RAS or PTPN11. Oncogene 2007; 26: 5816-5821

10 Fulda S, Vucic D. Targeting IAP proteins for therapeutic intervention in cancer. Nat RevDrug Discov 2012 im Druck

11 Fulda S, Wick W, Weller $M$ et al. Smac agonists sensitize for Apo2L/ TRAILor anticancer drug-induced apoptosis and induce regression of malignant glioma in vivo. Nat Med 2002; 8: 808-815 
12 Glocker E, Kotlarz D, Boztug $K$ et al. Inflammatory bowel disease and mutations affecting the IL10 receptor. New Engl J Med 2009; 361: 2033-2045

13 Göbel $U$, Fischer R, Henze G. From the therapy study to quality assurance in pediatric oncology. Klin Padiatr 1997; 209: 145-146

14 Göbel $U$, Witt $O$. Pitfalls of clinical registries in pediatric oncology and hematology. Klin Padiatr 2008; 220: 129-133

15 Göbel U. One group ticket "from bench to bedside", return, please!. Klin Padiatr 2005; 217: 307-309

16 Jumaa $H$, Bossaller L, Portugal $K$ et al. Deficiency of the adaptor SLP65 in pre-B-cell acute lymphoblastic leukaemia. Nature 2003; 423: 452-456

17 Jürgens $H$, Klingebiel T, Göbel $U$ et al. Supporting research in pediatric oncology and hematology. Klin Padiatr 2007; 219: 125-126

18 Klingebiel T, Creutzig $U$, Christaras A et al. Milestones of health policy and rare diseases. Klin Padiatr 2010; 222: 121-123

19 Klingebiel T, Creutzig U, Henze $G$ et al. Kind-Philipp Symposium in honor to the trustor Dr--Ing. Walter Reiners and Kind-Philipp Award 2008. Klin Padiatr 2009; 221: 327-331

20 Locatelli F, Nöllke P, Zecca $M$ et al.on behalf of the European Working Group of Childhood MDS (EWOG-MDS) and the European Blood and Marrow Transplantation (EBMT) Group. Hematopoietic stem cell transplantation (HSCT) in children with juvenile myelomonocytic leukemia (JMML): results of the EWOG-MDS/EBMT trial. Blood 2005; 105: 410-419

21 Loeder S, Fakler M, Schoeneberger $H$ et al. RIP is required for IAP inhibitor-mediated sensitization of childhood acute leukemia cells to chemotherapy induced apoptosis. Leukemia 2011 Dec 16. doi: 10.1038 lev.2011.353 [Epub ahead of print]

22 Loeder S, Zenz T, Schnaiter A et al. A novel paradigm to trigger apoptosis in chronic lymphocytic leukaemia. Cancer Res 2009; 69: 8977-8986

23 Meinhardt A, Burkhardt B, Zimmermann $M$ et al. Berlin-FrankfurtMünster group. Phase II window study on rituximab in newly diagnosed pediatric mature B-cell non-Hodgkin's lymphoma and Burkitt leukemia. J Clin Oncol 2010; 28: 3115-3121
24 Meyer LH, Eckhoff SM, Queudeville M et al. Early relapse in pediatric B cell precursor ALL is identified by Time To Leukemia (TTL) in NOD/ SCID mice and is characterized by a gene signature involving survival pathways. Cancer Cell 2011; 19: 206-2017

25 Meyer LH, Queudeville M, Eckhoff SM et al. Intact apoptosis signaling in myeloid leukaemia cells determines treatment outcome in childhood AML. Blood 2008; 111: 2899-2903

26 Niemeyer CM, Kang MW, Shin DH et al. Germline CBL mutations cause developmental abnormalities and predispose to juvenile myelomonocytic leukemia. Nat Genet 2010; 42: 794-800

27 Salzburg J, Burkhardt B, Zimmermann $M$ et al. Prevalence, clinical pattern, and outcome of CNS involvement in childhood and adolescent non-Hodgkin's lymphoma differ by non-Hodgkin's lymphoma subtype: a Berlin-Frankfurt-Munster Group Report. J Clin Oncol 2007; 25: 3915-3922

28 Schwermann J, Rathinam C, Schubert $M$ et al. MAPKAP kinase MK2 maintains self-renewal capacity of haematopoietic stem cells. EMBO J 2009; 28: 1392-1406

29 Strauss $G$, Lindquist JA, Arhel $N$ et al. CD95 co-stimulation blocks activation of naive $T$ cells by inhibiting $\mathrm{T}$ cell receptor signaling. J Exp Med 2009; 206: 1379-1393

30 Tartaglia $M^{*}$, Niemeyer $C^{*}$, Song $X$ et al. Somatic PTPN11 mutations in juvenile myelomonocytic leukemia, myelodysplastic syndromes and acute myeloid leukemia. Nat Genet 2003; 34: 148-150 ( ${ }^{*}$ Indicates authors who contributed equally to work)

31 Vogler $M$, Walczak $H$, Stadel $D$ et al. Small molecule XIAP inhibitors enhance TRAIL-induced apoptosis and antitumor activity in preclinical models of pancreatic carcinoma. Cancer Res 2009; 69: 2425-2434

32 Woessmann W, Zimmermann $M$, Lenhard $M$ et al. Relapsed or refractory anaplastic large-cell lymphoma in children and adolescents after Berlin-Frankfurt-Muenster (BFM)-type first-line therapy: a BFMgroup study. J Clin Oncol 2011; 29: 3065-3071 